\title{
On General Integral Operator of Analytic Functions
}

\author{
Nasser Alkasbi and Maslina Darus \\ School of Mathematical Sciences, Faculty of Science and Technology, Universiti Kebangsaan Malaysia, 43600 Bangi, Selangor, Malaysia
}

Correspondence should be addressed to Maslina Darus; maslina@ukm.my

Received 3 August 2013; Accepted 27 October 2013

Academic Editor: Mohamed Kamal Aouf

Copyright (c) 2013 N. Alkasbi and M. Darus. This is an open access article distributed under the Creative Commons Attribution License, which permits unrestricted use, distribution, and reproduction in any medium, provided the original work is properly cited.

Let $E_{\beta}$ be the integral operator defined by $E_{\beta}(z)=\left[\beta \int_{0}^{z} t^{\beta-1}\left(f_{1}^{\prime}(t)\right)^{\alpha_{1}}\left(f_{1}(t) / t\right)^{\gamma_{1}} P_{1}^{\zeta_{1}(t)} \cdots\left(f_{n}^{\prime}(t)\right)^{\alpha_{n}}\left(f_{n}(t) / t\right)^{\gamma_{n}} P_{n}^{\zeta_{n}(t)} d t\right]^{1 / \beta}$, where each of the functions $f_{i}$ and $P_{i}$ is, respectively, analytic functions and functions with positive real part defined in the open unit disk for all $i=1, \ldots, n$. The object of this paper is to obtain several univalence conditions for this integral operator. Our main results contain some interesting corollaries as special cases.

\section{Introduction and Definitions}

Let $A$ denote the class of functions of the form

$$
f(z)=z+\sum_{n=2}^{\infty} a_{n} z^{n}
$$

which are analytic in the open unit $\operatorname{disk} U=\{z: z \in \mathscr{C}:|z|<$ $1\}: S=\{f \in A: f$ is univalent in $U\}$. Also, let $\mathscr{P}$ be the class of all functions which are analytic in $U$ and satisfy $P(0)=1$, $\mathfrak{R}\{P(z)\}>0$. Frasin and Darus [1] defined the family $B(\delta)$, $0 \leq \delta<1$, so that it consists of functions $f \in A$ satisfying the condition

$$
\left|\frac{z^{2} f^{\prime}(z)}{f^{2}(z)}-1\right|<1-\delta \quad(z \in U) .
$$

In this paper, we obtain new sufficient conditions for the univalence of the general integral operator $E_{\beta}(z)$ defined by

$$
E_{\beta}(z)=\left[\beta \int_{0}^{z} t^{\beta-1} \prod_{i=0}^{n}\left(f_{i}^{\prime}(t)\right)^{\alpha_{i}}\left(\frac{f_{i}(t)}{t}\right)^{\gamma_{i}} P_{i}^{\zeta_{i}(t)} d t\right]^{1 / \beta},
$$

where $\beta \in \mathscr{C}, \alpha_{i}, \gamma_{i}, \zeta_{i} \in \mathscr{C}, f_{i} \in A$, and $P_{i} \in \mathscr{P}$ for all $i=$ $1,2,3, \ldots n$

Here and throughout in the sequel, every multivalued functions is taken with the principal branch.
Remark 1. Note that the integral operator $E_{\beta}$ generalizes the following operators introduced and studied by several authors as follows.

(i) For $\alpha_{i}=0$, where $i=1, \ldots, n$, we obtain the integral operator

$$
N_{\beta}(z)=\left[\beta \int_{0}^{z} t^{\beta-1} \prod_{i=0}^{n}\left(\frac{f_{i}(t)}{t}\right)^{\gamma_{i}} P_{i}^{\zeta_{i}(t)} d t\right]^{1 / \beta},
$$

introduced and studied by Frasin [2].

(ii) For $\alpha_{i}=\gamma_{i}=0, i=1, \ldots, n$, we obtain the integral operator

$$
\mathscr{F}_{\beta}^{\zeta}\left(P_{i}\right)(z)=\left[\beta \int_{0}^{z} t^{\beta-1} \prod_{i=0}^{n} P_{i}^{\zeta_{i}(t)} d t\right]^{1 / \beta},
$$

introduced and studied by Frasin [3].

(iii) For $\zeta_{i}=0, i=1, \ldots, n$, we obtain the integral operator

$$
\begin{aligned}
\mathcal{F}_{\beta}^{\alpha_{i}, \gamma_{i}} & \left(f_{1}, f_{2}, \ldots, f_{n}\right)(z) \\
= & {\left[\beta \int_{0}^{z} t^{\beta-1} \prod_{i=0}^{n}\left(f_{i}^{\prime}(t)\right)^{\alpha_{i}}\left(\frac{f_{i}(t)}{t}\right)^{\gamma_{i}} d t\right]^{1 / \beta}, }
\end{aligned}
$$


(iv) For $\zeta_{i}=0, \beta=1$, and $i=1, \ldots, n$, we obtain the integral operator

$\mathscr{J}^{\alpha_{i}, \gamma_{i}}\left(f_{1}, f_{2}, \ldots, f_{n}\right)(z)=\int_{0}^{z} \prod_{i=0}^{n}\left(f_{i}^{\prime}(t)\right)^{\alpha_{i}}\left(\frac{f_{i}(t)}{t}\right)^{\gamma_{i}} d t$

introduced and studied by Frasin [5].

(v) For $\zeta_{i}=\alpha_{i}=0$ and $i=1, \ldots, n$, we obtain the integral operator

$\mathscr{I}_{\gamma}^{\beta_{i}}\left(f_{1}, f_{2}, \ldots, f_{n}\right)(z)$

$$
=\left[\beta \int_{0}^{z} t^{\beta-1}\left(\frac{f_{1}(t)}{t}\right)^{\gamma_{1}} \cdots\left(\frac{f_{n}(t)}{t}\right)^{\gamma_{n}} d t\right]^{1 / \beta},
$$

introduced and studied by D. Breaz and N. Breaz [6].

(vi) For $\beta=1, \zeta_{i}=\alpha_{i}=0$, and $i=1, \ldots, n$, we obtain the integral operator

$$
F(z)=\int_{0}^{z}\left(\frac{f_{1}(t)}{t}\right)^{\gamma_{1}} \cdots\left(\frac{f_{n}(t)}{t}\right)^{\gamma_{n}} d t
$$

introduced and studied by D. Breaz and N. Breaz [6].

(vii) For $\beta=1, \zeta_{i}=\gamma_{i}=0$, and $i=1, \ldots, n$, we obtain the integral operator

$$
F_{\alpha_{1}, \ldots, \alpha_{n}}(z)=\int_{0}^{z}\left(f_{1}^{\prime}(t)\right)^{\alpha_{1}} \cdots\left(f_{n}^{\prime}(t)\right)^{\alpha_{n}} d t
$$

introduced and studied by Breaz et al. [7].

(viii) For $\beta=1, n=1, \gamma_{1}=\gamma$, and $\alpha_{i}=\zeta_{i}=0$, we obtain the integral operator

$$
F_{\alpha, \gamma}(z)=\int_{0}^{z}\left(\frac{f(t)}{t}\right)^{\gamma}\left(f^{\prime}(t)\right)^{\alpha} d t
$$

studied in [8].

(ix) For $\beta=1, n=1, \gamma_{1}=\gamma$, and $\alpha_{i}=\zeta_{i}=0$, we obtain the integral operator

$$
F_{\gamma}(z)=\int_{0}^{z}\left(\frac{f(t)}{t}\right)^{\gamma} d t
$$

studied in [9]. In particular, for $\gamma=1$, we obtain Alexander integral operator which was introduced in [10] as follows

$$
F(z)=\int_{0}^{z} \frac{f(t)}{t} d t
$$

(x) For $\beta=1, n=1, \alpha_{i}=\alpha$, and $\gamma_{i}=\zeta_{i}=0$, we obtain the integral operator

$$
G(z)=\int_{0}^{z}\left(f^{\prime}(t)\right)^{\alpha} d t
$$

studied in [11].

In order to derive our main results, we have to recall here the following lemmas.
Lemma 2 (see [12]). Let $\eta \in \mathscr{C}$ with $\mathfrak{R}(\eta)>0$. If $h \in A$ satisfies

$$
\frac{1-|z|^{2 \Re(\eta)}}{\mathfrak{R}(\eta)}\left|\frac{z h^{\prime \prime}(z)}{h^{\prime}(z)}\right| \leq 1
$$

for all $z \in U$, then the integral operator

$$
F_{\beta}(z)=\left[\beta \int_{0}^{z} t^{\beta-1}\left(f^{\prime}(t)\right) d t\right]^{1 / \beta}
$$

is in the class $\mathcal{S}$.

Lemma 3 (see [13]). Let $\beta \in \mathscr{C}$ with $\mathfrak{R}(\beta)>0, c \in \mathscr{C}$ with $|c| \leq 1, c \neq-1$. If $h \in A$ satisfies

$$
\left.|c| z\right|^{2 \beta}+\left(1+|z|^{2 \beta}\right) \frac{z h^{\prime \prime}(z)}{\beta h^{\prime}(z)} \mid \leq 1
$$

for all $z \in U$, then the integral operator $F_{\beta}(z)$ defined by $(16)$ is in the class $\mathcal{S}$.

Lemma 4 (see $[14]$ ). If $P(z) \in \mathscr{P}$, then

$$
\left|\frac{z P^{\prime \prime}(z)}{\beta P^{\prime}(z)}\right| \leq \frac{2|z|}{1-|z|^{2}} .
$$

Lemma 5 (see [9]). If $f(z) \in B(\delta)$, then

$$
\left|\frac{z f^{\prime}(z)}{f(z)}-1\right| \leq \frac{(1-\delta)(1+|z|)}{1-|z|}
$$

When $\delta=0$, so $f \in \mathcal{S}$.

Lemma 6 (see [9]). If $f(z) \in B(\delta)$, then

$$
\left|\frac{z f^{\prime \prime}(z)}{f^{\prime}(z)}\right| \leq \frac{(1-\delta)(2+|z|)}{1-|z|} .
$$

Also we need the following general Schwarz lemma.

Lemma 7 (see [15]). Let the function $f$ be regular in the disk $U_{R}=\{z:|z|<R\}$, with $|M|<R$ for fixed $M$. If $f(z)$ has one zero with multiplicity order bigger than $m$ for $z=0$, then

$$
|f(z)| \leq \frac{M}{R^{m}}|z|^{m}, \quad\left(z \in U_{R}\right) .
$$

The equality holds only if

$$
f(z)=e^{i \theta}\left(\frac{M}{R^{m}}\right) z^{m},
$$

where $\theta$ is constant.

Lemma 8 (see [16]). If $f \in A$, then

$$
\left|1+\frac{z f^{\prime \prime}(z)}{f^{\prime}(z)}\right| \leq \frac{5}{4}\left|\frac{z f^{\prime}(z)}{f(z)}\right| .
$$




\section{Univalence Conditions for the Operator $E_{\beta}$}

We first prove the following theorem.

Theorem 9. Let $f \in B\left(\delta_{i}\right), 0 \leq \delta_{i}<1, P_{i}(z) \in \mathscr{P}$ for all $i=1, \ldots, n$ and $\eta \in \mathscr{C}$ with $\mathfrak{R}(\eta)=a>0$. If

$$
\sum_{i=1}^{n}\left[\left(3\left|\alpha_{i}\right|+2\left|\gamma_{i}\right|\right)\left(1-\delta_{i}\right)+2\left|\zeta_{i}\right|\right]<\min \left\{a ; \frac{1}{2}\right\}
$$

then the integral operator $E_{\beta}$ defined by (3) is in the class $\mathcal{S}$.

Proof. Define the regular function $h(z)$ by

$$
h(z)=\int_{0}^{z} \prod_{i=0}^{n}\left[\left(f_{i}^{\prime}(t)\right)^{\alpha_{i}}\left(\frac{f_{i}(t)}{t}\right)^{\gamma_{i}} P_{i}^{\zeta_{i}(t)}\right] d t .
$$

Then it is easy to see that

$$
h^{\prime}(z)=\prod_{i=0}^{n}\left[\left(f_{i}^{\prime}(t)\right)^{\alpha_{i}}\left(\frac{f_{i}(t)}{t}\right)^{\gamma_{i}} P_{i}^{\zeta_{i}(t)}\right],
$$

and $h(0)=1-h^{\prime}(0)=0$. Differentiating both sides of $(26)$ logarithmically, we obtain

$$
\begin{aligned}
\frac{z h^{\prime \prime}(z)}{h^{\prime}(z)}= & \sum_{i=1}^{n} \alpha_{i}\left(\frac{z f_{i}^{\prime \prime}(z)}{f_{i}^{\prime}(z)}\right)+\sum_{i=1}^{n} \gamma_{i}\left(\frac{z f_{i}^{\prime}(z)}{f_{i}(z)}-1\right) \\
& +\sum_{i=1}^{n} \zeta_{i}\left(\frac{z P_{i}^{\prime}(z)}{P_{i}(z)}\right) .
\end{aligned}
$$

Thus, we have

$$
\begin{aligned}
\left|\frac{z h^{\prime \prime}(z)}{h^{\prime}(z)}\right| \leq & \sum_{i=1}^{n}\left|\alpha_{i}\right|\left|\frac{z f_{i}^{\prime \prime}(z)}{f_{i}^{\prime}(z)}\right|+\sum_{i=1}^{n}\left|\gamma_{i}\right|\left|\frac{z f_{i}^{\prime}(z)}{f_{i}(z)}-1\right| \\
& +\sum_{i=1}^{n}\left|\zeta_{i}\right|\left|\frac{z P_{i}^{\prime}(z)}{P_{i}(z)}\right| .
\end{aligned}
$$

Since $f_{i} \in B\left(\delta_{i}\right), P_{i}(z) \in \mathscr{P}$ for all $i=1, \ldots, n$, from (28), (18), (19), and (20), we obtain

$$
\begin{aligned}
\left|\frac{z h^{\prime \prime}(z)}{h^{\prime}(z)}\right| & \\
\leq & \sum_{i=1}^{n}\left|\alpha_{i}\right|\left(1-\delta_{i}\right)\left(\frac{2+|z|}{1-|z|}\right) \\
& +\sum_{i=1}^{n}\left|\gamma_{i}\right|\left(1-\delta_{i}\right)\left(\frac{1+|z|}{1-|z|}\right)+\sum_{i=1}^{n}\left|\zeta_{i}\right|\left(\frac{2|z|}{1-|z|^{2}}\right) \\
\leq & \sum_{i=1}^{n}\left|\alpha_{i}\right|\left(1-\delta_{i}\right)\left(\frac{3}{1-|z|}\right) \\
& +\sum_{i=1}^{n}\left|\gamma_{i}\right|\left(1-\delta_{i}\right)\left(\frac{2}{1-|z|}\right)+\sum_{i=1}^{n}\left|\zeta_{i}\right|\left(\frac{2}{1-|z|}\right) .
\end{aligned}
$$

Multiplying both sides of (29) by $\left(1-|z|^{2 \Re(\eta)}\right) / \mathfrak{R}(\eta)$, we get

$$
\begin{aligned}
\frac{1-|z|^{2 \mathfrak{R}(\eta)}}{\mathfrak{R}(\eta)}\left|\frac{z h^{\prime \prime}(z)}{h^{\prime}(z)}\right| \\
\leq \frac{1-|z|^{2 \mathfrak{R}(\eta)}}{(1-|z|) \Re(\eta)} \\
\quad \times \sum_{i=1}^{n}\left[\left(3\left|\alpha_{i}\right|+2\left|\gamma_{i}\right|\right)\left(1-\delta_{i}\right)+2\left|\zeta_{i}\right|\right],
\end{aligned}
$$

for all $z \in U$.

Let us denote $|z|=x, x \in[0,1), \mathfrak{R}(\eta)=a>0$, and $\Phi(x)=\left(1-x^{2 a}\right) /(1-x)$. It is easy to prove that

$$
\Phi(x) \leq \begin{cases}1, & 0<a<1, \\ 2 a, & \frac{1}{2}<a<\infty .\end{cases}
$$

From (31), (30), and the hypotheses (24), we have

$$
\begin{aligned}
& \frac{1-|z|^{2 a}}{a}\left|\frac{z h^{\prime \prime}(z)}{h^{\prime}(z)}\right| \\
& \leq\left\{\begin{array}{cl}
\frac{1}{a} \sum_{i=1}^{n}\left[\left(3\left|\alpha_{i}\right|+2\left|\gamma_{i}\right|\right)\left(1-\delta_{i}\right)+2\left|\zeta_{i}\right|\right], & 0<a<1, \\
2 \sum_{i=1}^{n}\left[\left(3\left|\alpha_{i}\right|+2\left|\gamma_{i}\right|\right)\left(1-\delta_{i}\right)+2\left|\zeta_{i}\right|\right], & \frac{1}{2}<a<\infty
\end{array}\right.
\end{aligned}
$$$$
\leq 1 \text {, }
$$

for all $z \in U$. Applying Lemma 2 for the function $h(z)$, we prove that $E_{\beta}(z) \in \mathcal{S}$.

Letting $n=1, \delta_{1}=\delta, \alpha_{1}=\alpha, \gamma_{1}=\gamma$, and $\zeta_{1}=\zeta$ in Theorem 9, we obtain the following corollary.

Corollary 10. Let $f \in B(\delta), 0 \leq \delta<1, P(z) \in \mathscr{P}$, and all $\eta, \gamma, \zeta \in \mathscr{C}$ with $\Re(\eta)=a>0$. If

$$
(3|\alpha|+2|\gamma|)(1-\delta)+2|\zeta|<\min \left\{a ; \frac{1}{2}\right\},
$$

and then the integral operator $N_{\beta}^{\alpha, \gamma, \zeta}$ defined by

$$
N_{\beta}^{\alpha, \gamma, \zeta}(z)=\left[\beta \int_{0}^{z} t^{\beta-1}\left(f^{\prime}(t)\right)^{\alpha}\left(\frac{f(t)}{t}\right)^{\gamma} P^{\zeta}(t) d t\right]^{1 / \beta}
$$

is in the class $\mathcal{S}$.

If we set $\delta=0$ in Corollary 10, we have the following.

Corollary 11. Let $f \in \mathcal{S}, P(z) \in \mathscr{P}$ and all $\eta, \gamma, \zeta \in \mathscr{C}$ with $\mathfrak{R}(\eta)=a>0$. If

$$
3|\alpha|+2|\gamma|+2|\zeta|<\min \left\{a ; \frac{1}{2}\right\},
$$

then the integral operator $N_{\beta}^{\alpha, \gamma, \zeta}$ defined by (34) is in the class $\delta$. 
Next, we prove the following theorem.

Theorem 12. Let $\alpha_{i}, \gamma_{i}, \zeta_{i} \in \mathscr{C}$ for all $i=1, \ldots, n$ and each $f_{i} \in A$ satisfies condition (15) with $\mathfrak{R}\left(f_{i}(z) / z\right)>0$ and

$$
\sum_{i=1}^{n}\left[29\left|\alpha_{i}\right|+16\left|\gamma_{i}+\zeta_{i}\right|\right] \leq \begin{cases}4 \Re(\eta), & \text { if } \mathfrak{R}(\eta) \in(0,1), \\ 4, & \text { if } \mathfrak{R}(\eta) \in[1, \infty)\end{cases}
$$

and then, for any complex number $\sigma$ with $\mathfrak{R}(\sigma) \geq \mathfrak{R}(\eta)>0$, the integral operator $N_{\beta}$ defined by (4) is in the class $\mathcal{S}$.

Proof. Suppose that $\mathfrak{R}\left(f_{i}(z) / z\right)$ for all $i=1, \ldots, n$. Thus we have

$$
\frac{f_{i}(z)}{z}=P_{i}(z)
$$

where $P_{i} \in \mathscr{P}$ for all $i=1, \ldots, n$. Differentiating both sides of (37) logarithmically, we obtain

$$
\frac{z f_{i}^{\prime}(z)}{f_{i} z}-1=\frac{z P_{i}^{\prime}(z)}{P_{i}(z)}
$$

Define the regular function $h(z)$ as in (25). Thus from (27) we have

$$
\frac{z h^{\prime \prime}(z)}{h^{\prime}(z)}=\sum_{i=1}^{n} \alpha_{i}\left(\frac{z f_{i}^{\prime \prime}(z)}{f_{i}^{\prime}(z)}\right)+\sum_{i=1}^{n}\left(\gamma_{i}+\zeta_{i}\right)\left(\frac{z f_{i}^{\prime}(z)}{f_{i}(z)}-1\right)
$$

and so

$$
\begin{aligned}
\frac{z h^{\prime \prime}(z)}{h^{\prime}(z)}= & \sum_{i=1}^{n} \alpha_{i}\left(\frac{z f_{i}^{\prime \prime}(z)}{f_{i}^{\prime}(z)}+1\right)-\sum_{n=i}^{0} \alpha_{i} \\
& +\sum_{i=1}^{n}\left(\gamma_{i}+\zeta_{i}\right)\left(\frac{z f_{i}^{\prime}(z)}{f_{i}(z)}-1\right) .
\end{aligned}
$$

From Lemma 8, it follows that

$$
\begin{aligned}
\left|\frac{z h^{\prime \prime}(z)}{h^{\prime}(z)}\right| \leq & \frac{5}{4} \sum_{i=1}^{n}\left|\alpha_{i}\right|\left|\frac{z f_{i}^{\prime}(z)}{f_{i}(z)}\right| \\
& +\sum_{i=1}^{n}\left|\gamma_{i}+\zeta_{i}\right|\left|\frac{z f_{i}^{\prime}(z)}{f_{i}(z)}-1\right|+\sum_{n=i}^{0}\left|\alpha_{i}\right| \\
\leq & \frac{5}{4} \sum_{i=1}^{n}\left|\alpha_{i}\right|\left[\left|\frac{z f_{i}^{\prime}(z)}{f_{i}(z)}-1\right|+1\right] \\
& +\sum_{i=1}^{n}\left|\gamma_{i}+\zeta_{i}\right|\left|\frac{z f_{i}^{\prime}(z)}{f_{i}(z)}-1\right|+\sum_{n=i}^{0}\left|\alpha_{i}\right| \\
\leq & \sum_{i=1}^{n}\left[\left(\frac{5}{4}\left|\alpha_{i}\right|+\left|\gamma_{i}+\zeta_{i}\right|\right)\left|\frac{z f_{i}^{\prime}(z)}{f_{i}(z)}-1\right|\right] \\
& +\frac{9}{4} \sum_{i=1}^{n}\left|\alpha_{i}\right|
\end{aligned}
$$

$$
\begin{aligned}
\leq & \sum_{i=1}^{n}\left[\left(\frac{5}{4}\left|\alpha_{i}\right|+\left|\gamma_{i}+\zeta_{i}\right|\right)\left(\left|\frac{z f_{i}^{\prime}(z)}{f_{i}(z)}\right|+1\right)\right] \\
& +\frac{9}{4} \sum_{i=1}^{n}\left|\alpha_{i}\right| .
\end{aligned}
$$

Multiplying both sides of (41) by $\left(1-|z|^{2 \Re(\eta)}\right) / \Re(\eta)$, from Lemma 5 with $\delta=0$, we get

$$
\begin{aligned}
& \frac{1-|z|^{2 \mathfrak{R}(\eta)}}{\mathfrak{R}(\eta)}\left|\frac{z h^{\prime \prime}(z)}{h^{\prime}(z)}\right| \\
& \leq \frac{1-|z|^{2 \mathfrak{R}(\eta)}}{\mathfrak{R}(\eta)} \sum_{i=1}^{n}\left(\frac{5}{4}\left|\alpha_{i}\right|+\left|\gamma_{i}+\zeta_{i}\right|\right)\left(\frac{2}{1-|z|}\right) \\
&+\frac{9\left(1-|z|^{2 \mathfrak{R}(\eta)}\right) \sum_{i=1}^{n}\left|\alpha_{i}\right|}{4 \Re(\eta)} .
\end{aligned}
$$

Suppose that $\mathfrak{R}(\eta) \in(0,1)$. Define a function $\Phi:(0,1) \rightarrow R$ by

$$
\Phi(x)=1-a^{2 a} \quad(0<a<1) .
$$

Then $\Phi$ is an increasing function and consequently, for $|z|=$ $a ; z \in U$, we obtain

$$
1-|z|^{2 \Re(\eta)}<1-|z|^{2}
$$

We thus find from (42) and (44) that

$$
\begin{aligned}
\frac{1-|z|^{2 \Re(\eta)}}{\mathfrak{R}(\eta)}\left|\frac{z h^{\prime \prime}(z)}{h^{\prime}(z)}\right| \leq & \frac{\sum_{i=1}^{n}\left(5\left|\alpha_{i}\right|+4\left|\gamma_{i}+\zeta_{i}\right|\right)}{\Re(\eta)} \\
& +\frac{9 \sum_{i=1}^{n}\left|\alpha_{i}\right|}{4 \Re(\eta)} \\
= & \frac{\sum_{i=1}^{n}\left(29\left|\alpha_{i}\right|+19\left|\gamma_{i}+\zeta_{i}\right|\right)}{4 \Re(\eta)} .
\end{aligned}
$$

Using the hypotheses (36) for $\mathfrak{R}(\eta) \in(0,1)$, we readily get

$$
\frac{1-|z|^{2 \Re(\eta)}}{\Re(\eta)}\left|\frac{z h^{\prime \prime}(z)}{h^{\prime}(z)}\right| \leq 1 .
$$

Now if $\mathfrak{R}(\eta) \in[1, \infty)$, we define a function $\Psi:[1, \infty) \rightarrow R$ by

$$
\Psi(x)=\frac{1-a^{2 x}}{x}, \quad(0<a<1) .
$$

We observe that the function $\Psi$ is decreasing and consequently, for $|z|=a ; z \in U$, we have

$$
\frac{1-|z|^{2 \Re(\eta)}}{\Re(\eta)}<1-|z|^{2}
$$

for all $z \in U$. It follows from (40) and (42) that

$$
\frac{1-|z|^{2 \Re(\eta)}}{\Re(\eta)}\left|\frac{z h^{\prime \prime}(z)}{h^{\prime}(z)}\right| \leq \sum_{i=1}^{n}\left(\frac{29}{4}\left|\alpha_{i}\right|+4\left|\gamma_{i}+\zeta_{i}\right|\right) .
$$


Using once again the hypotheses (36) when $\mathfrak{R}(\eta) \in[1, \infty)$, we easily get

$$
\frac{1-|z|^{2 \Re(\eta)}}{\mathfrak{R}(\eta)}\left|\frac{z h^{\prime \prime}(z)}{h^{\prime}(z)}\right| \leq 1
$$

Finally by applying Lemma 2, we conclude that the integral operator $N_{\beta}$ defined by (4) is in the class $\delta$.

Letting $n=1, \delta_{1}=\delta, \alpha_{1}=\alpha, \gamma_{1}=\gamma$, and $\zeta_{1}=\zeta$ in Theorem 12, we obtain the following corollary.

Corollary 13. Let $\alpha, \gamma, \zeta \in \mathscr{C}$, and $f \in A$ satisfies condition (15). If

$$
29|\alpha|+16|\gamma+\zeta| \leq \begin{cases}4 \mathfrak{R}(\eta), & \text { if } \mathfrak{R}(\eta) \in(0,1), \\ 4, & \text { if } \mathfrak{R}(\eta) \in[1, \infty)\end{cases}
$$

then, for any complex number $\sigma$ with $\mathfrak{R}(\sigma) \geq \mathfrak{R}(\eta)>0$, the integral operator $N_{\beta}^{\alpha, \gamma, \zeta}$ defined by (4) is in the class $\delta$.

Using Lemma 3, we derive the following theorem.

Theorem 14. Let $\alpha_{i}, \gamma_{i}, \zeta_{i}, \beta \in \mathscr{C}$ for all $i=1, \ldots, n, \mathfrak{R}(\beta)>0$, $c \in \mathscr{C}(|c| \leq 1)$ and each $f_{i} \in A$ satisfies condition (15). If

$$
\sum_{i=1}^{n}\left[29\left|\alpha_{i}\right|+16\left|\gamma_{i}+\zeta_{i}\right|\right] \leq \begin{cases}4 \beta(1-|c|), & \text { if } \mathfrak{R}(\eta) \in(0,1), \\ 4(1-|c|), & \text { if } \mathfrak{R}(\eta) \in[1, \infty)\end{cases}
$$

then, for any complex number $\sigma$ with $\mathfrak{R}(\sigma) \geq \mathfrak{R}(\eta)>0$, the integral operator $N_{\beta}$ defined by (4) is in the class $\mathcal{S}$.

Proof. From (40), we have

$$
\begin{aligned}
& \left.|c| z\right|^{2 \beta}+\left(1-|z|^{2 \beta}\right) \frac{z h^{\prime \prime}(z)}{\beta h^{\prime}(z)} \mid \\
& =\left.|c| z\right|^{2 \beta}+\frac{1-|z|^{2} \beta}{\beta} \\
& \quad \times\left[\sum_{i=1}^{n} \alpha_{i}\left(\frac{z f_{i}^{\prime \prime}(z)}{f_{i}^{\prime}(z)}+1\right)-\sum_{i=1}^{n} \alpha_{i}\right. \\
& \left.\quad+\sum_{i=1}^{n}\left(\gamma_{i}+\zeta_{i}\right)\left(\frac{z f_{i}^{\prime}(z)}{f_{i}(z)}-1\right)\right] \mid \\
& \quad \times\left[\sum_{i=1}^{n}\left(\frac{5}{4}\left|\alpha_{i}\right|+\left|\gamma_{i}+\zeta_{i}\right|\right)\left(\left|\frac{z f_{i}^{\prime}(z)}{f_{i}(z)}\right|+1\right)+\frac{9}{4} \sum_{i=1}^{n}\left|\alpha_{i}\right|\right] \\
& \leq|c|+\frac{\left.|1-| z\right|^{2} \mid}{|\beta|} \\
& \quad \times\left[\sum_{i=1}^{n}\left(\frac{5}{4}\left|\alpha_{i}\right|+\left|\gamma_{i}+\zeta_{i}\right|\right)\left(\frac{2}{1-|z|}\right)+\frac{9}{4} \sum_{i=1}^{n}\left|\alpha_{i}\right|\right] .
\end{aligned}
$$

Suppose that $\beta \in(0,1)$. Define a function $\Phi:(0,1) \rightarrow R$ by

$$
\Phi(x)=1-a^{2 x} \quad(0<a<1) .
$$

Then $\Phi$ is an increasing function and consequently for $|z|=$ $a ; z \in U$, we obtain

$$
1-|z|^{2 \beta}<1-|z|^{2} .
$$

We thus find from (53) that

$$
\left.|c| z\right|^{2 \beta}+\left(1-|z|^{2 \beta}\right) \frac{z h^{\prime \prime}(z)}{\beta h^{\prime}(z)} \mid \leq \frac{\sum_{i=1}^{n}\left(29\left|\alpha_{i}\right|+16\left|\gamma_{i}+\zeta_{i}\right|\right)}{4|\beta|} .
$$

Using the hypotheses (52) for $\beta \in(0,1)$, we readily get

$$
\left.|c| z\right|^{2 \beta}+\left(1-|z|^{2 \beta}\right) \frac{z h^{\prime \prime}(z)}{\beta h^{\prime}(z)} \mid \leq 1 .
$$

Now if $\beta \in[1, \infty)$, we define a function $\Psi:[1, \infty) \rightarrow R$ by

$$
\Psi(x)=\frac{1-a^{2 x}}{x}, \quad(0<a<1) .
$$

We observe that the function $\Psi$ is decreasing and consequently for $|z|=a ; z \in U$, and using once again the hypotheses (36) when $\mathfrak{R}(\eta) \in[1, \infty)$, we easily get

$$
\left.|c| z\right|^{2 \beta}+\left(1-|z|^{2 \beta}\right) \frac{z h^{\prime \prime}(z)}{\beta h^{\prime}(z)} \mid \leq 1 .
$$

Finally, by applying Lemma 3, we conclude that $N_{\beta} \in \mathcal{S}$.

\section{Conflict of Interests}

The authors declare that they have no conflict interests.

\section{Authors' Contribution}

The first author is currently a Ph.D. student under supervision of the second author and jointly worked on deriving the results. All authors read and approved the paper.

\section{Acknowledgment}

The work presented here was partially supported by ERGS/1/ 2013/STG06/UKM/01/2 and DIP-2013-1.

\section{References}

[1] B. A. Frasin and M. Darus, "On certain analytic univalent functions," International Journal of Mathematics and Mathematical Sciences, vol. 25, no. 5, pp. 305-310, 2001.

[2] B. A. Frasin, "General integral operator of analytic functions involving functions with positive real part," Journal of Mathematics, vol. 2013, Article ID 260127, 4 pages, 2013.

[3] B. A. Frasin, "Integral operator of analytic functions with positive real part," Kyungpook Mathematical Journal, vol. 51, no. 1, pp. 77-85, 2011. 
[4] B. A. Frasin, "Order of convexity and univalency of general integral operator," Journal of the Franklin Institute, vol. 348, no. 6, pp. 1013-1019, 2011.

[5] B. A. Frasin, "New general integral operator," Computers and Mathematics with Applications, vol. 62, no. 11, pp. 4272-4276, 2011.

[6] D. Breaz and N. Breaz, "Two integral operators, Studia Universitatis Babes-Bolyai," Mathematica, vol. 3, pp. 13-21, 2002.

[7] D. Breaz, S. Owa, and N. Breaz, "A new integral univalent operator," Acta Universitatis Apulensis, no. 16, pp. 11-16, 2008.

[8] M. Dorf and J. Szynal, "Linear invariance and integral operators of univalent functions," Demonstratio Mathematica, vol. 38, no. 1, pp. 47-57, 2005.

[9] E. Deniz and H. Orhan, "An extension of the univalence criterion for a family of integral operators," Annales Universitatis Mariae Curie-Sklodowska A, vol. 64, no. 2, pp. 29-35, 2010.

[10] W. Alexander, "Functions which map the interior of the unit circle upon simple regions," Annals of Mathematics, vol. 17, no. 1, pp. 12-22, 1915.

[11] N. Pascu and V. Pescar, "On the integral operators of KimMerkes and Pfaltzgraff," Mathematica, vol. 32, no. 2, pp. 185-192, 1990.

[12] N. Pascu and V. Pescar, “An improvement of Becker's univalence criterion," in Proceedings of the Commemorative Session: Simion Stoilow (Brasov 1987) (Brasov), pp. 43-48, University of Brasov, 1987.

[13] V. Pescar, "Univalence of certain integral operators," Acta Universitatis Apulensis, vol. 12, no. 2006, pp. 43-48, 1989.

[14] T. H. MacGregor, "The radius of univalence of certain analytic functions," Proceedings of the American Mathematical Society, vol. 14, pp. 514-520, 1963.

[15] Z. Nehari, Conformal Mapping, Dover, New York, NY, USA, 1975.

[16] M. Obradovic and S. Owa, "A criterion for starlikeness," Mathematische Nachrichten, vol. 140, pp. 97-102, 1978. 


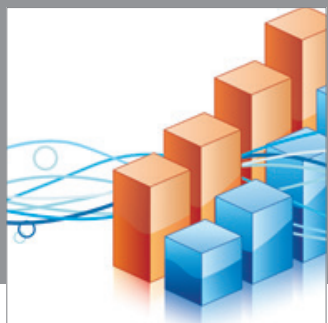

Advances in

Operations Research

mansans

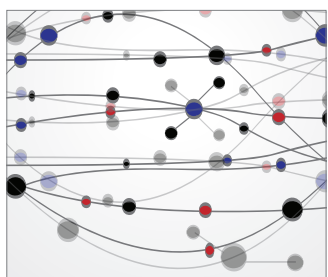

The Scientific World Journal
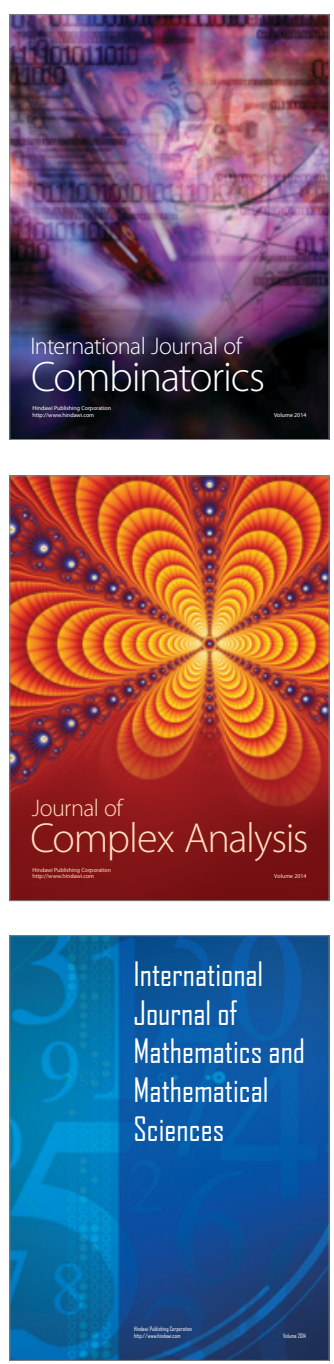
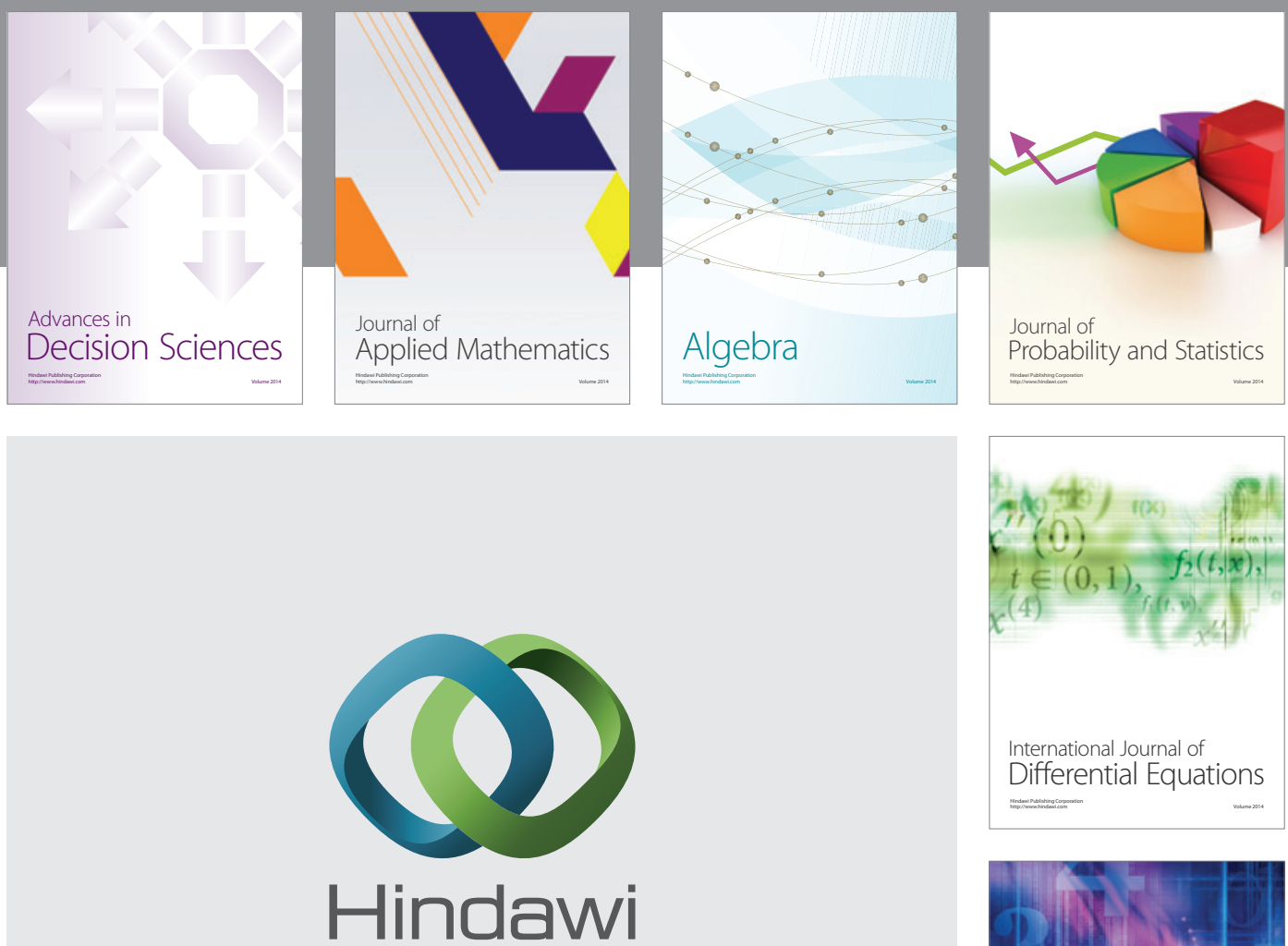

Submit your manuscripts at http://www.hindawi.com
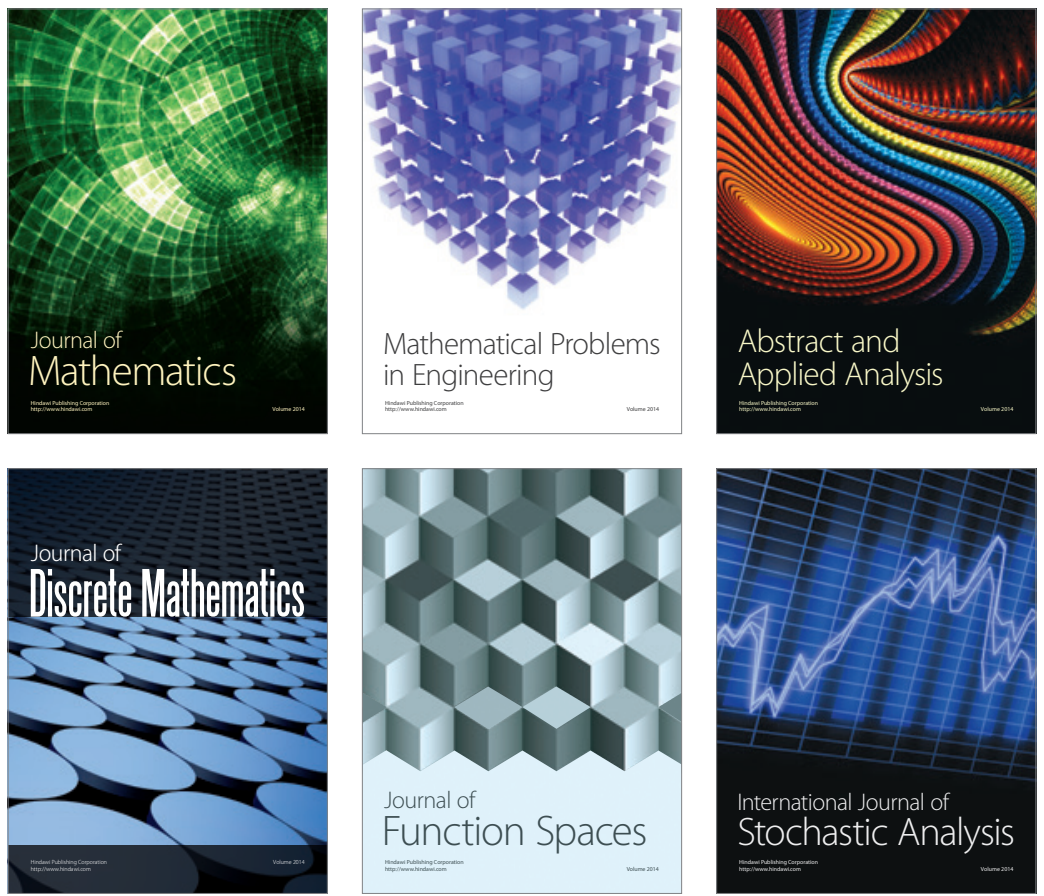

Journal of

Function Spaces

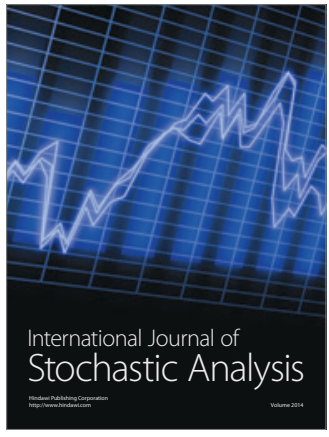

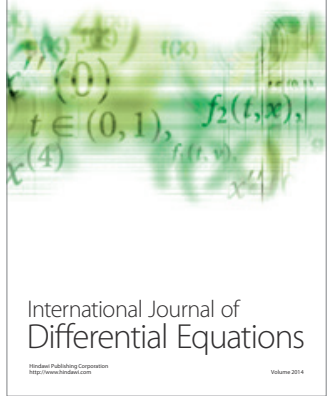
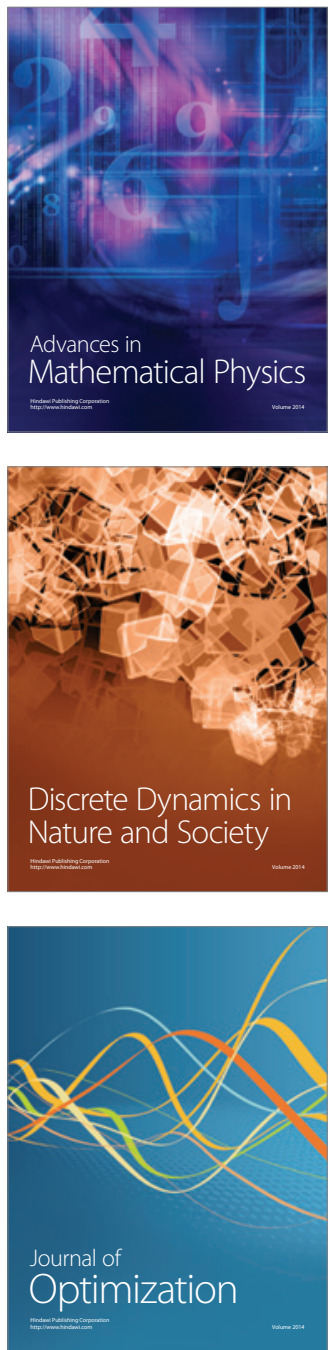